\title{
Reading paradigms: From lab to cyberspace?
}

\author{
DORIS AARONSON and EDWARD COLET \\ New York University, New York, New York
}

\begin{abstract}
Computer-controlled display procedures useful for doing reading research are discussed. The procedures used in the present research vary the text unit size (from single words to half-sentences), the amount of context available (no context and varying amounts of prior text), text movement (centered words or successive left-to-right placement), and use of text highlighting (present, absent). The data suggest links between the display attributes and the subjects' coding strategies during reading of mathematical word problems. Display attributes can influence the time course of processing, the subjectively coded unit size, and the nature of linguistic processing. The data also have implications for display attributes that may make reading in cyberspace more user friendly for both novice and expert readers.
\end{abstract}

The use of lab computers to control text displays for reading research has spawned several new research paradigms. Some of these paradigms have human factors attributes that may make them useful for selected realworld applications in educational contexts, including cyberspace, which will provide much of the future education for both children and adults. Perhaps the most frequently used is the RSVP (rapid serial visual presentation) procedure (Potter, Kroll, \& Harris, 1980; Potter \& Levy, 1969), in which individual words (or pictures) are presented very rapidly and sequentially in the center of a monitor with the primary data being subjects' error rates in serial or free recall tasks. Although this procedure might be useful for training speed readers (Potter et al., 1980 ), it does not take advantage of the computer's interactive capabilities (e.g., subject-controlled display rate).

In the 1970s, more flexible "word-by-word" reading procedures were developed (Aaronson \& Scarborough, 1976, 1977) that permitted subjects to pace themselves through a word sequence that formed a sentence. With each subject-paced keypress, the reading time (RT) was recorded for the prior word (centered on the screen), which was then overwritten by the next word. After the last word, a variety of response tasks could be used, including recall, recognition, comprehension, or grammaticality judgments. In 1982, Just, Carpenter, and Woolley renamed the wordby-word procedure the "fixed-window" or "stationary window" procedure and contrasted it with a "moving window" procedure, in which the screen was initially filled with dashes in positions of nonspace characters. After the first displayed word, each subject-paced keypress replaced the prior word by dashes and displayed the next word in the position to the right, thus moving line by line

\footnotetext{
We thank Thomas M. Donnelly, Michael Palij, Sarah Ransdell, and Jennifer B. Thomas for helpful comments. This research was supported in part by PHS Grant MH-16,496 from the National Institutes of Health to D.A. Correspondence should be addressed to D. Aaronson, Psychology Department, Room 860, New York University, 6 Washington Pl., New York, NY 10003 (e-mail: doris@psych. nyu.edu).
}

through a paragraph. Just et al. also used a "cumulative" condition in which keypresses displayed each new word, but all preceding words remained on the screen.

These three classes of display procedures have attributes that may lead to differing cognitive strategies. The moving-window and the cumulative condition permit subjects to make relatively normal eye movements (including regressions) and to have "preview" and "postview" from peripheral vision of the physical structure and, for the cumulative procedure, of the linguistic content of any prior text. But as research tools, these two procedures have some problems. Keypresses do not necessarily reflect the RT for the current word, and also the stimulus field increases in size as more and more text is displayed. Just et al. (1982) reported that subjects "sometimes used a strategy of rapidly pushing the response button three or four times in succession and then reading the entire group of newly presented words" (p. 230). In contrast, the fixedwindow or word-by-word procedure requires subjects to focus their attention on the single word displayed at the moment, and it also eliminates time-consuming eye movements to other text locations. This provided research advantages for interpreting RTs for particular words and their linguistic attributes.

The present research was designed in part to explore and expand further some of the attributes of computersupported reading paradigms, as well as their impact on reading strategies for good and poor readers. In particular, variations in display attributes may lead to differences in (1) the temporal course of processing, including eye movements; (2) the subjective unit size encoded for short-term memory; and (3) the nature of linguistic processing, including semantic integration and redundancy elimination (see Aaronson \& Ferres, .1986, for a review of past evidence for such reading strategy differences). Since past research indicates advantages for "local" (word-level) processing with the word-by-word display, but advantages for "global" processing with the cumulative display, a paradigm combining those features could be effective for reading. Our development of the moving-highlight pro- 
cedures is an attempt to do this. Further, one would expect that good subjects ("experts") would be more sensitive to display variations than poor subjects (relative "novices").

\section{METHOD}

\section{Procedure}

We selected three computer-based reading paradigms (described below) that provided a wide range of display unit sizes. All three were developed using the MEL programming language (Schneider, 1988) on a Zeos 386 PC with a Zeos 14-in. black-and-white flat-screen monitor. Sixty dark-adapted college students each experienced all three displays, one in each of three sessions, in a blocked design, with the order of blocks counterbalanced over subjects. Stimulus sentences had matched linguistic attributes for the three display types, with each sentence being a mathematical problem. These 16-word sentences, along with their questions, required 1,2 , or 3 addition or multiplication operations for solution (matched across display types). The seven-word questions, with all words displayed simultaneously on one line, preceded each sentence, and subjects typed their answers on a numeric keypad following each sentence. Text characters were in white on a black background and were $3 \mathrm{~cm}$ wide by $.8 \mathrm{~cm}$ tall. For each display type there were 5 practice and 48 experimental trials. Two sample stimuli were as follows: Question: "How many feet long was the fence?" Sentence: "The white fence stretched 91 feet across the yard then 3 feet back to the house." (Answer: 94). Question: "How many glasses did Tom break altogether?" Sentence: "Tom broke 2 boxes of 6 glasses per box 2 times on each of 3 days." (Answer: 72).

\section{Display Paradigms}

Figure 1 illustrates what we now call the "center-word" display, which used the word-by-word procedure for the 16-word sentences. Using the zero key on the numeric keypad, subjects displayed a trial prompt and then a question. After that, each time the subject pressed the zero key, a new word from the sentence appeared in the center of the screen, the previous word was erased, and its RT from the preceding keypress was recorded. Thus, no eye movements were required, and the RT could be attributed to processing during the time that the given word was displayed. This provides a direct way to obtain RTs as a function of word attributes such as length, frequency in the vocabulary, lexical category (e.g., noun), syntactic role (e.g., phrase break), mathematical role (e.g., number), and so on.

For the half-sentence display, shown in the middle of Figure 1, after the trial prompt and the subject-displayed question, the first 7-9 words were displayed. This unit ended at a major phrase boundary, and all words appeared on a single line. Following the subject's zero keypress, the first half-sentence was overwritten by the second half. For this procedure, RTs for word attributes can be indexed statistically by regression analysis (see Haberlandt, Graesser, \& Schneider, 1989). The half-sentence display provides a situation that is fairly close to normal reading, permitting eye movements, preview and postview, and a normal line length, requiring a long left saccade to the next line.

The moving-highlight procedure (Figure 1, bottom) differed from the previous paradigms in a few ways. Each zero keypress displayed a new highlighted word (i.e., printed in black, within a white boxed area). This highlighted word appeared to the right of previous words, which were then unhighlighted. Only 4 previous words

\section{CENTER WORD}

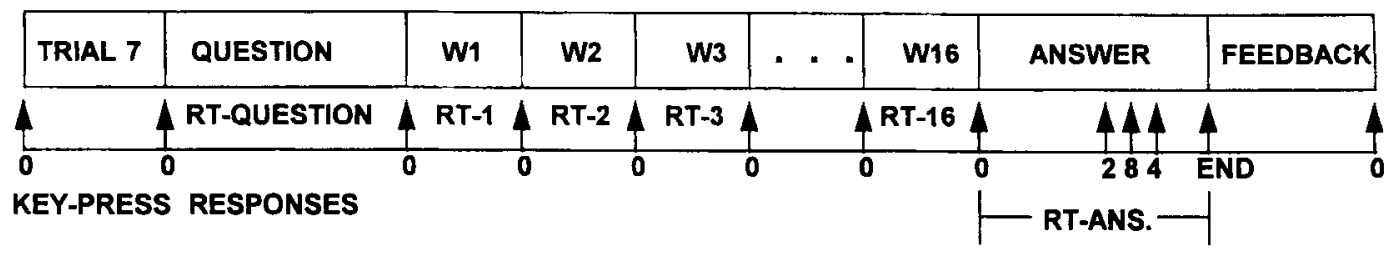

HALF-SENTENCE

\begin{tabular}{|c|c|c|c|c|c|}
\hline TRIAL 7 & QUESTION & W1 - W8 & W9 - W16 & ANSWER & FEEDBACK \\
\hline & RT-QUESTION & RT- 1ST HALF & RT- 2ND HALF & & \\
\hline 0 & & & & 284 & END \\
\hline \multicolumn{4}{|c|}{ KEY-PRESS RESPONSES } & RT-ANS. & \\
\hline
\end{tabular}

\section{MOVING HIGHLIGHT}

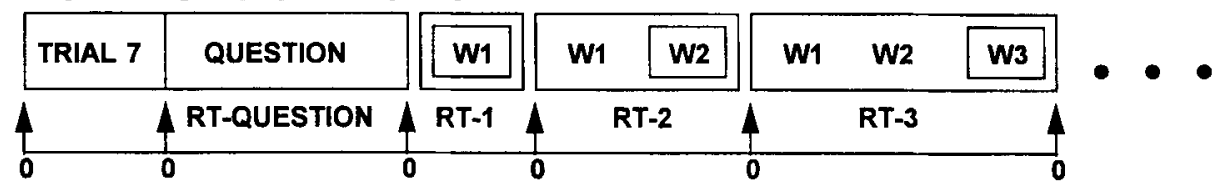

KEY-PRESS RESPONSES

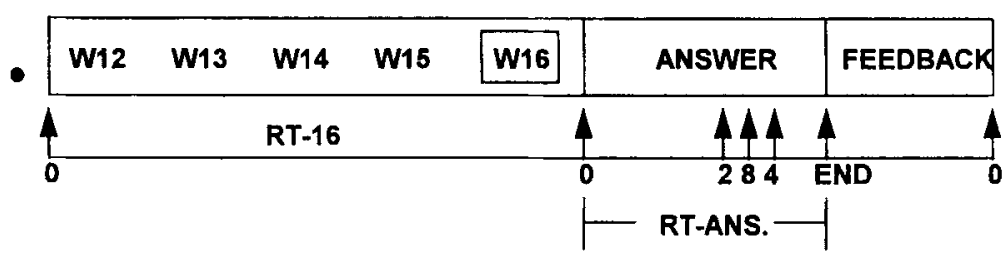

Figure 1. Illustrations of three text display paradigms that vary in unit size, prior context, movement, and highlighting. 
remained on the screen, with words beyond that being erased. The first 8 words were displayed on a central line, with the next 8 being displayed on the line below. The moving-highlight procedure has some of the positive attributes of both the center-word and halfsentence displays. As is the case for the center-word display, highlighting of the newest word does a good job of keeping the subject's focus on that particular word: Subjects' keypress RTs generally appear to reflect the linguistic attributes of the highlighted word (e.g., longer RTs for longer or lower frequency words). However, as is the case for the half-sentence display, some additional text is still available with each new highlighted word. The previous 4 (unhighlighted) words still on the screen are generally within subjects' span of peripheral vision, should they need to refresh that mental representation. But these previous words are restricted in both amount and location, in contrast to Just et al.'s (1982) continuous display, which retains all prior text.

If we consider the links between the three displays and cognitive/linguistic reading strategies, (1) the center-word display encourages subjects to devote maximum attention to the single newest word on the screen; (2) the half-sentence display provides subjects with the best opportunity to integrate information from a large amount of text including the possibility of regressive saccades; and (3) the moving-highlight display permits a flexible compromise between local and global information processing. Highlighting enhances word-level processing; in addition, the 4 prior words are generally within the perceptual and cognitive spans, which facilitates "chunking" into linguistic phrase units of an appropriate size for short-term memory.

To evaluate the effects of the three display paradigms on reading strategies, we will focus on four performance measures: (1) the average RT per word, (2) the time to answer the questions, (3) the accuracy in answering those questions, and (4) the time to read the initial question, which may be linked to strategies for reading the following sentence.

\section{RESULTS}

\section{Paradigm Effects}

Now let us consider some data relevant to the three display formats. Figure $2 \mathrm{~A}$ shows the average RT per word for each display type. RTs are ordered inversely with the display unit size [main effect of displays: $F(2,59)=$ $11.81, p<.01]$. Subjects spent about 60 msec more per word with the moving-highlight than with the half-sentence display, and they spent another $100 \mathrm{msec}$ more for the center-word display. The center-word display may promote excessive coding of 16 small memory units, whereas half-sentences may reduce coding through semantic integration and elimination of redundant or irrel-
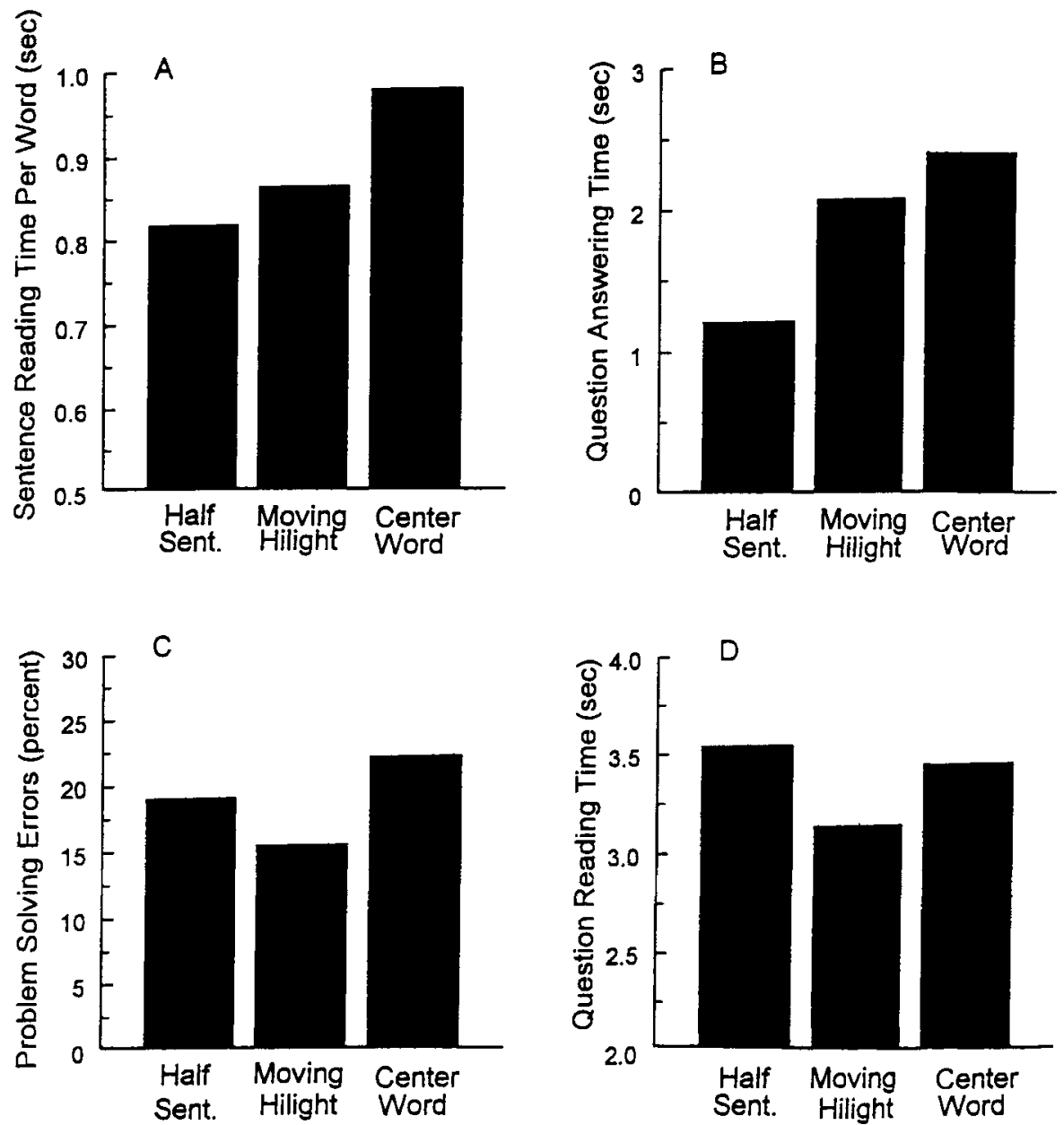

Figure 2. Response data for three text display paradigms. (A) Sentence-reading times. (B) Questionanswering times. (C) Problem-solving errors. (D) Question-reading times. 
evant text. The moving-highlight display yields a compromise between such local and global coding for memory. Note that the RT differences cannot be attributed to differing numbers of keypresses for the various conditions, because the center-word and moving-highlight procedures both require a keypress for each word. Further, previous research with the center-word display indicates that keypresses likely occur in parallel with higher level processing (Aaronson \& Ferres, 1986; Aaronson \& Scarborough, 1977).

Figure 2B shows the mean answering times for each display type. Answering times include both computing time beyond that done while reading and typing time for numerical keypress responses. These answering times are ordered in the same way as the initial word-reading times [main effect of displays: $F(2,59)=42.44, p<.01$ ] Subjects spent almost $1 \mathrm{sec}$ more with the moving-highlight display format than with the half-sentence format, and yet another $1 / 3 \mathrm{sec}$ with the center-word format. The displays with faster correct answering times may have less text to be retrieved from memory and processed during the answering period, because of earlier semantic integration and elimination of redundant or irrelevant text.

However, the error data in Figure $2 \mathrm{C}$ provide some surprises. Subjects made the fewest errors with the movinghighlight display and the most errors with the centerword format, with a difference of almost $7 \%$ between them relative to an average for the three display formats of $18.7 \%$ [main effect of displays: $F(2,59)=19.34, p<$ $.01]$. Why might the moving-highlight format have yielded the best accuracy? First, the center-word condition may load up working memory with 16 small units, leaving insufficient capacity for text integration or on-line computing. Next, the half-sentences, in which redundant or irrelevant text is often eliminated, may lead to disaster if that text later turns out to be critical to problem solution. The moving-highlight format, however, provides an optimal compromise: linguistic phrase-sized memory units may provide for some text integration, and less text elimination than with the half-sentence format. Also, these medium-sized units may leave more memory capacity free for computing than that which occurs in the centerword format, thus yielding higher accuracy rates. Support for a tradeoff between number of memory units and unit size has been provided by Yntema and Mueser (1960).

The RTs for the initial problem questions in Figure 2D provide some data consistent with the above rationale. The moving-highlight format not only yielded the fewest errors, but it also had the fastest question RTs-over $400 \mathrm{msec}$ faster than the half-sentence format [main effects of displays: $F(2,59)=4.59, p<.02$ ]. The question comes first and is displayed in the same way for all conditions. But that question must be well coded to survive the 16-unit memory-load of the center-word displays and also to guide text elimination with the half-sentence displays. Again, the moving-highlight format is a compro- mise: With both less memory stress than with centerword displays and less text elimination than with halfsentence displays, questions are read quickly.

\section{Subject Effects}

Now let us briefly consider some data on differences between good and poor subjects for the three display procedures. These data are shown in Figure 3. Good and poor readers were defined by being in either the top or the bottom third of our 60 subjects, based on mean problemsolving accuracy for the three display types. Thus, the interesting questions concern interactions between reader groups and display types.

Figures $3 \mathrm{~A}$ and $3 \mathrm{~B}$ show that the rank order of display formats was generally similar for good and poor subjects for both sentence RTs and question RTs. Namely, conditions with larger display units were read and answered more quickly because of semantic integration and elimination of redundant or irrelevant text, as noted earlier for the total data pool. The interesting interaction is that good subjects did a better job of adjusting their sentence RTs to the display types (Figure 3A), with an RT difference of about $200 \mathrm{msec}$ per word between the fastest and slowest conditions, in comparison to about half that difference for poor subjects [group $\times$ display interaction: $F(2,76)=2.46, p<.09$ ]. A consequence of their failure to allocate time adequately to the more difficult input conditions, poor subjects exhibited greater differences between conditions in answering times than did good subjects (Figure 3B), although the group $\times$ display interaction was not significant. The difference between the slowest and fastest mean answering times was over $400 \mathrm{msec}$ greater for poor than for good subjects.

Figures $3 \mathrm{C}$ and $3 \mathrm{D}$ exhibit analogous interactions between subject groups and display formats to those in Figures $3 \mathrm{~B}$ and $3 \mathrm{~A}$. Because good subjects appropriately slowed down their sentence RTs for the smaller display unit sizes (Figure 3A), their hardest display condition suffered very little in accuracy (Figure $3 \mathrm{C}$ ). However, poor subjects, who did not adequately adapt their sentence RTs to the display conditions (Figure 3A), consequently had large differences in their resulting accuracy: about a $10 \%$ difference between the center-word and moving-highlight displays, as compared with only $2.7 \%$ for the good subjects (Figure 3C) [group $\times$ display interaction: $F(2,76)=4.04, p<.02]$.

Lastly, the analysis yielded the expected interaction between subject groups and display formats for the initial question RTs (Figure 3D). Good subjects appropriately allocated more question RTs for the half-sentence format, with its text-elimination strategy, and for the center-word format, with its heavy memory load. Although the group $X$ display interaction was not significant ( $n$ was only 20 per group for Figure 3), poor subjects showed smaller differences among conditions for their question RTs $(380 \mathrm{msec})$ in relation to $600 \mathrm{msec}$ for good subjects. 

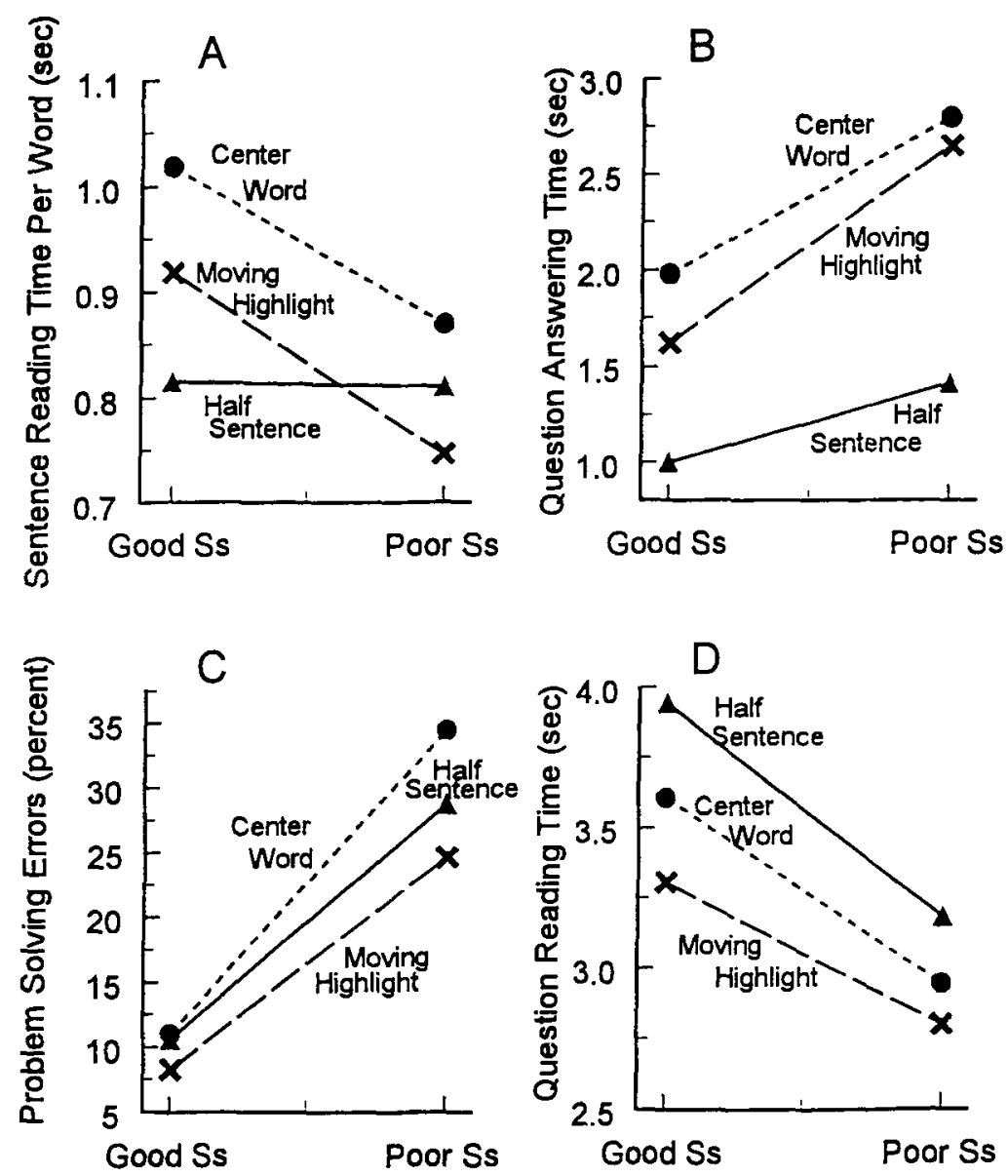

Figure 3. Response data for subjects with "good" and "poor" accuracy for three text display paradigm. (A) Sentence-reading times. (B) Question-answering times. (C) Problemsolving errors. (D) Question-reading times.

\section{IMPLICATIONS FOR EDUCATION AND CYBERSPACE}

Our data indicate that the computer display format influences the performance of good and poor subjects, both the input text-processing times for the problem questions and sentences and the output times and accuracies for problem solution. With the Internet and World-Wide Web providing information and education for both adults and children, including the requirement to read and comprehend text displays, psychologists and educators should be studying and developing ways to optimize those reading experiences.

The present research suggests that two display attributes may be important to optimize performance in cyberspace. First, the moving-highlight feature appears to facilitate performance because it helps focus the reader's attention, thereby reducing unnecessary regressive eye movements, and, especially in the case of children and some poor readers, including dyslexics, helps them keep their place in a large body of text. Both novice child readers and many dyslexics frequently lose their place in a large body of text. Thus, they are taught to move their finger along each line as they read from books, as a place keeper. When one is reading in cyberspace, being able to move a highlighted or color-coded block across the words, using the arrow keys or a mouse, would be a better option than using one's finger. Further, preprogrammed highlighting could use color or brightness to indicate text attributes to the reader (perhaps based on menu selections), such as relevance or importance of the text to a preselected topic area. The moving-highlight display format yielded higher performance accuracy than did the other two displays, indicating that it might optimize the educational value of cyberspace, especially for learning in children.

Second, given individual differences among good and poor readers, more flexible options for unit sizes to step through text displays may be needed. Instead of just one line or one screen at a time, readers could select a text unit size that is optimal for their own memory span and reading skills and for the difficulty of the given text content and/or task demands. Newcomers to the system might select from a menu of reading abilities or task dif- 
ficulties to obtain a small, medium, or large unit size by clicking on low, average, or high reading ability (or possibly school grade) or task difficulty (e.g., in-depth comprehension, skim for gist, or topic search). Experienced users might directly select their preferred unit size in terms of number of words. Finally, one might provide text- and task-based questions periodically, and, depending on the reader's accuracy, do one of the following: (1) have the computer adjust the unit size or (2) provide feedback to the user to do so. Web-browser software is approaching sophistication levels at which these types of applications could in fact be implemented. Psychologists and educators, however, would need to do further research to determine which types of options would be most effective for the user and the type of reading task.

However, a larger class of concerns for educators and cognitive psychologists focuses on the content rather than the format of Web information - namely, the extent to which cyberspace will develop as a strong educational source or as a source for amusement. Hopefully, some psychologists will provide research that indicates how to use the amusement-relevant attributes of a multimedia environment to enrich the educational experience. Without proper research, the fun and games could well end up as costly and time-consuming distractions that could turn yet more people into "couch potatoes." Cognitive scientists involved in basic research should be building more bridges to move their research findings into appli- cations that can benefit human information processing in the real world.

\section{REFERENCES}

Aaronson, D., \& Ferres, S. (1986). Reading strategies for children and adults: A quantitative model. Psychological Review, 93, 89-112. Aaronson, D., \& SCARBOROUGH, H. (1976). Performance theories for sentence coding: Some quantitative evidence. Journal of Experimental Psychology: Human Perception \& Performance, 2, 56-70.

aAronson, D., \& Scarborough, H. (1977). Performance theories for sentence coding: Some quantitative models. Journal of Verbal Learning \& Verbal Behavior, 16, 277-303.

Haberlandt, K. F., Graesser, A. C., \& Schneider, N. J. (1989). Reading strategies of fast and slow readers. Journal of Experimental Psychology: Learning, Memory, \& Cognition, 15, 815-823.

Just, M. A., Carpenter, P. A., \& Woolley, J. D. (1982). Paradigms and processes in reading comprehension. Journal of Experimental Psychology: General, 111, 228-238.

PotTer, M. C., Kroll, J. F., \& Harris, C. (1980). Comprehension and memory in rapid serial processing. In R. Nickerson (Ed.), Attention and performance VIII (pp. 395-418). Hillsdale, NJ: Erlbaum.

PotTer, M. C., \& Levy, E. I. (1969). Recognition memory for a rapid sequence of pictures. Journal of Experimental Psychology, 81, 10-15.

SCHNEIDER, W. (1988). Micro Experimental Laboratory: An integrated system for IBM PC compatibles. Behavior Research Methods, Instruments, \& Computers, 20, 206-217.

YNTEMA, D. B., \& Mueser, G. (1960). Remembering the present state of a number of variables. Journal of Experimental Psychology, 60, 18-22.

(Manuscript received October 3, 1996; revision accepted for publication February 12, 1997.) 